\title{
FAST MEDICAL DIAGNOSTICS USING AUTOASSOCIATIVE NEURO-FUZZY MEMORY
}

\author{
Iryna Perova, Yevgeniy Bodyanskiy \\ Kharkiv National University of Radio Electronics, 14 Nauky av., Kharkiv, 61166 Ukraine \\ rikywenok@gmail.com, yevgeniy.bodyanskiy@nure.ua
}

\begin{abstract}
This paper proposes an architecture of fast medical diagnostics system based on autoassociative neuro-fuzzy memory. The architecture of proposed system is close to traditional Takagi-Sugeno-Kang neuro-fuzzy system, but it is based on other principles. This system contains of recording subsystem and pattern retrieval subsystem, where diagnostics of patients with unknown diagnoses is realized. Level of memberships for all other possible diagnoses from recording subsystem is determined too. System tuning is based on lazy learning procedure and "neurons in data points" principle and uses bell-shaped fuzzy basis functions. Number of these functions changes during training process using principles of evolving connectionist systems. Bell-shaped membership functions centers can be tuned using proposed algorithm, processes of accumulation patients in fundamental memory and patients retrieval are described. This hybrid neuro-fuzzy associative memory combines advantages of fuzzy inference systems, artificial neural networks and evolving systems and its using provides the increasing of autoassociative memories capacity without essential complication of its architecture for medical diagnostics tasks. Copyright $\mathbb{C}$ Research Institute for Intelligent Computer Systems, 2017. All rights reserved.
\end{abstract}

Keywords: medical diagnostics, medical data mining, computational intelligence, kernel fuzzy basis function, autoassociative neuro-fuzzy memory, neuro-fuzzy system.

\section{INTRODUCTION}

In medical diagnostics tasks, physicians often use such properties of the human brain to remember medical features and later to retrieve them using associations with any other medical cases. That means that an autoassociative system can identify a patient who has been introduced to the system before through some time. This property can help diagnose any rare diseases that were detected by this physician earlier and there is no need any statistic or other cases of this disease to be found. Associative memory neural networks (AMNN) are the class of artificial neural networks, which replicates this human brain function [1-6]. Hopfield neural network, eigenvector automata, correlation matrixmemory, BSB-neural networks, bidirectional associative memory are the most used of AMNN [7]. All these memories have the common disadvantage - small memory volume, which is determined by the $l / n$ value, where $l$ is recording patient number, $n$ - their dimensionality.

There is interesting class of associative memory networks called LAMN - associative memory networks based on lattice structures $[8,9]$ that are similar by architecture to RBF (Radial Basis
Functions) Networks. Those networks possess a larger capacity but have principal disadvantage exponential growth of neurons number in hidden layer with growth of input vector dimensionality

So, hybrid neuro-fuzzy systems [11,12] became the one of preferred approaches because they combine the advantages of fuzzy inference systems and artificial neural networks, provide the increasing of autoassociative memories volume without complication of its structure.

\section{LEARNING OF AUTOASSOCIATIVE MEMORY USING BELL-SHAPED UNIVARIATE FUNCTIONS}

The neuro-fuzzy architecture for autoassociative memory tasks is presented on Fig.1 and contains four layers of information processing. It is easy to see that proposed system is similar to L.WangJ.Mendel neuro-fuzzy system in its architecture $[10,13]$. Also it may solve a problems of accumulation the fundamental memory patients data set

$$
x^{*}(k) \in R^{n}, k=1,2, \ldots, l,
$$


that can be presented in the form of matrix $X^{*}$ with dimensionality $(n \times l)$.

The first layer realizes the membership functions $\mu_{\tilde{x}_{i}^{*}(k)}$ that correspond to the $j$-th coordinate of $k$-th accumulated patient and consist of $n l$ nodes.

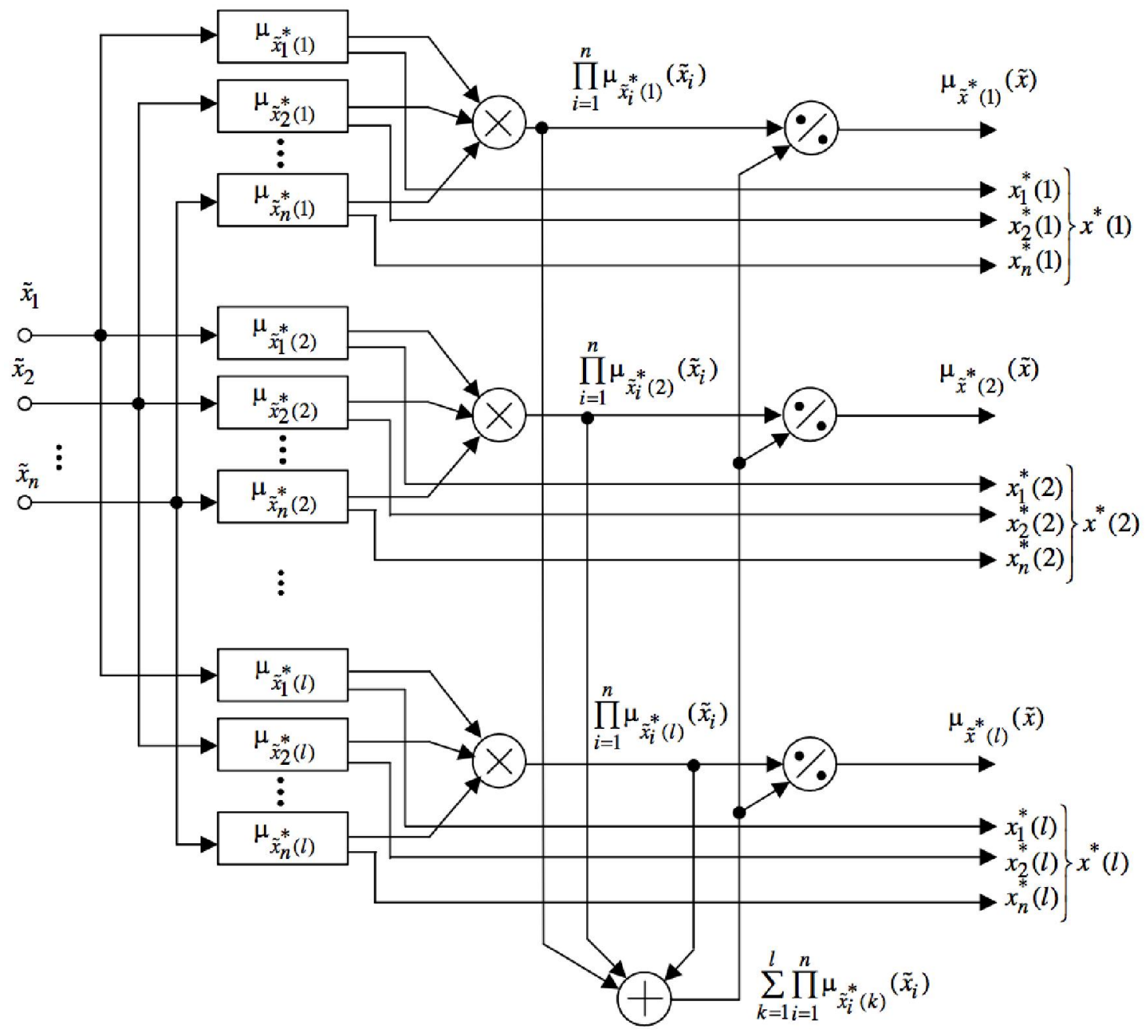

Fig. 1 - Architecture of associative neuro-fuzzy memory

In [17] the membership function presented in the form of triangle construction, but in medical diagnostics tasks that functions must have infinite support region for protection of "gaps" in fuzzyficated space. In situation when diagnosisclasses can be overlapped bell-shaped membership function with infinite support is shown on Fig. 2 and can de described by analytical expression:

$$
\mu_{l i}\left(\tilde{x}_{i}\right)=e^{-\left(\frac{\tilde{x}_{i}-c_{l i}}{\sigma}\right)^{2 b}}
$$

where $c_{l i}-$ center parameter of membership function.

In situation when membership function centers irregularly cover input space it would be advisable to tune their width using parameter $\sigma$. The goal of this tuning is make sure that two neighboring function cross in same level that all others ones (parameter $\Delta$ on Fig. 2).

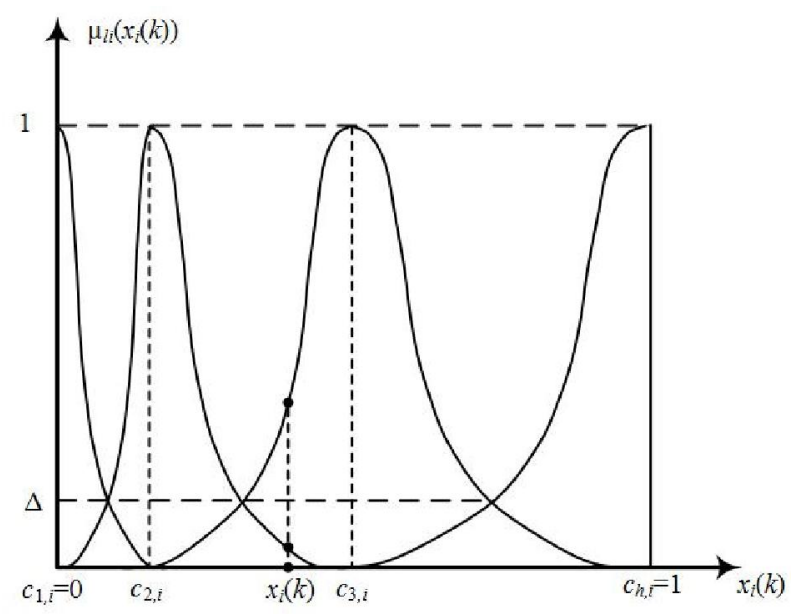

Fig. 2 - Bell-shaped membership function 
It is easy to see that membership functions are encoded on the interval from 0 to 1 . So processed medical data set should be defined on the same interval using the expression

$$
\tilde{x}_{i}^{*}=\frac{x_{i}^{*}-x_{i}^{* \min }}{x_{i}^{*}{ }^{* \max }-x_{i}^{* \min }}
$$

where $x_{i}^{* \min }$ and $x_{i}^{* \max }$ are minimal and maximal value of $i$-th raw elements. Expressing from (2) variable $x_{i}^{*}$ :

$$
x_{i}^{*}=x_{i}^{* \max } \tilde{x}_{i}^{*}-x_{i}^{* \min }\left(\tilde{x}_{i}^{*}-1\right) .
$$

It follows, that if $x_{i}^{* \min } \leq x_{i}^{*} \leq x_{i}^{* \max }$ then $0 \leq \tilde{x}_{i}^{*} \leq 1$.

Functions of membership (1) integrated in $l$ blocks with $n$ elements in every one, and each of formed blocks coincide to one patient with specific diagnosis from fundamental memory.

At second hidden layer $n$ membership functions proceed to $l$ multiplication blocks, where the aggregation of each stored patients is implemented. In output of second hidden layer a values $\prod_{i=1}^{n} \mu_{\tilde{x}_{i}^{*}(k)}\left(\tilde{x}_{i}\right)$ are computed. At third hidden layer aggregated values of membership functions are added in the summation, where the value $\sum_{k=1}^{l} \prod_{i=1}^{n} \mu_{\tilde{x}_{i}^{*}(k)}\left(\tilde{x}_{i}\right)$ is computed. At last layer (output layer) the scalability of the signal is realized. Thus, when the arbitrary medical features vector $\tilde{x}$ of dimensionality $n$ is fed to the system input, in the system output $l$ signals $\mu_{\tilde{x}_{i}(k)}(\tilde{x})$ are appear.

$$
\mu_{\tilde{x}_{i}(k)}(\tilde{x})=\frac{\prod_{i=1}^{n} \mu_{\tilde{x}_{i}^{*}(k)}\left(\tilde{x}_{i}\right)}{\sum_{k=1}^{l} \prod_{i=1}^{n} \mu_{\tilde{x}_{i}^{*}(k)}\left(\tilde{x}_{i}\right)}, k=1,2, \ldots, l
$$

A principle "neurons at data points" uses for learning process of the proposed architecture [14]. That means that at the first layer centers of the membership functions are agree with the coordinates of accumulated patient projections. This learning algorithm is similar to learning of the general regression neural networks [15] and probabilistic neural networks [16].
As a result, the architecture of the associative neuro-fuzzy memory is build and this architecture can be presented in the form of recording subsystem associative neuro-fuzzy memory [17]. In the output of proposed subsystem membership functions which are determined the association with early known disease are calculated.

\section{PATTERN RETRIEVAL FROM THE NEURO-FUZZY AUTOASSOCIATIVE MEMORY}

Architecture presented on Fig. 1 can be used for patients retrieval when all $l$ patients $\tilde{x}^{*}(1), \tilde{x}^{*}(2)$, $\ldots, \tilde{x}^{*}(l)$ with specific diagnoses are accumulated in the autoassociative memory. This architecture must be supplemented by any blocks, shown on Fig. 3: maximum detector, multiplier units and adder witch contain $n l$ inputs. All next patients $x(p)$, $p=l+1, l+2, \ldots$, must be defined using (2) before feeding to input of the system. But situations when $\tilde{x}_{i}(p)$ takes value differing from interval $[0 ; 1]$ can happens. That's why we need to add a transformation of the input signals for it view in the form of unit segment from 0 to 1 using expression:

$$
\tilde{x}_{i}(p)=\left\{\begin{array}{l}
0, \quad \text { if } \quad \tilde{x}_{i}(p)<0 \\
\tilde{x}_{i}(p), \quad \text { if } \quad 0 \leq \tilde{x}_{i}(p) \leq 1 \\
1, \quad \text { if } \quad \tilde{x}_{i}(p)>1
\end{array}\right.
$$

When information about new patient presented in the form of vector $\tilde{x}(p)$ goes to the system input (Fig.3), its membership levels to each of the patient with specific diagnosis from the fundamental memory are determined as

$\mu_{\tilde{x}^{*}(k)}(\tilde{x}(p))=\frac{\prod_{i=1}^{n} \mu_{\tilde{x}_{i}^{*}(k)}\left(\tilde{x}_{i}(p)\right)}{\sum_{k=1}^{l} \prod_{i=1}^{n} \mu_{\tilde{x}_{i}^{*}(k)}\left(\tilde{x}_{i}(p)\right)}, k=1,2, \ldots, l$.

After that, maximal membership value $\mu_{\tilde{x}^{*}(k)}^{\max }(\tilde{x}(p))$ is defined in maximum detector and in the autoassociative neuro-fuzzy memory output signals we can see maximal value of membership function and patient with specific diagnosis $\tilde{x}^{*}(k)=\arg \mu_{\tilde{x}^{*}(k)}^{\max }(\tilde{x}(p))$ corresponding to it. 


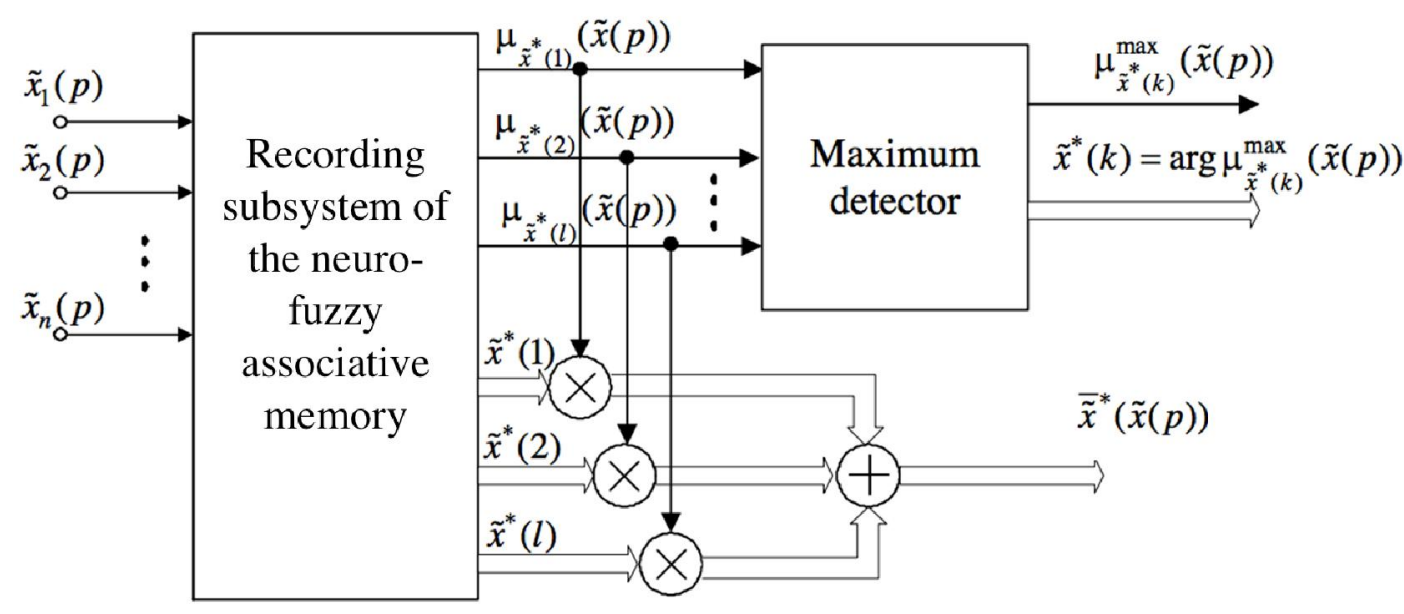

Fig. 3 - Patterns retrieval in neuro-fuzzy associative memory

Proposed system can provide association of processed new patient $\tilde{x}(p)$ with all other ones, represented in the fundamental memory $\tilde{x}^{*}(k)$, that was connected by nonzero membership level. So, the "hybrid" patient, presented as a combination of the specific patients of the fundamental memory emerge in the system output

$$
\overline{\tilde{x}}^{*}(\tilde{x}(p))=\frac{\sum_{k=1}^{l} \mu_{\tilde{x}^{*}(k)}(\tilde{x}(p)) \tilde{x}^{*}(k)}{\sum_{k=1}^{l} \mu_{\tilde{x}^{*}(k)}(\tilde{x}(p))} .
$$

Attraction regions of fundamental memory patients $\tilde{x}^{*}(1), \tilde{x}^{*}(2), \tilde{x}^{*}(3)$ are presented in Fig.4 like hatched areas.

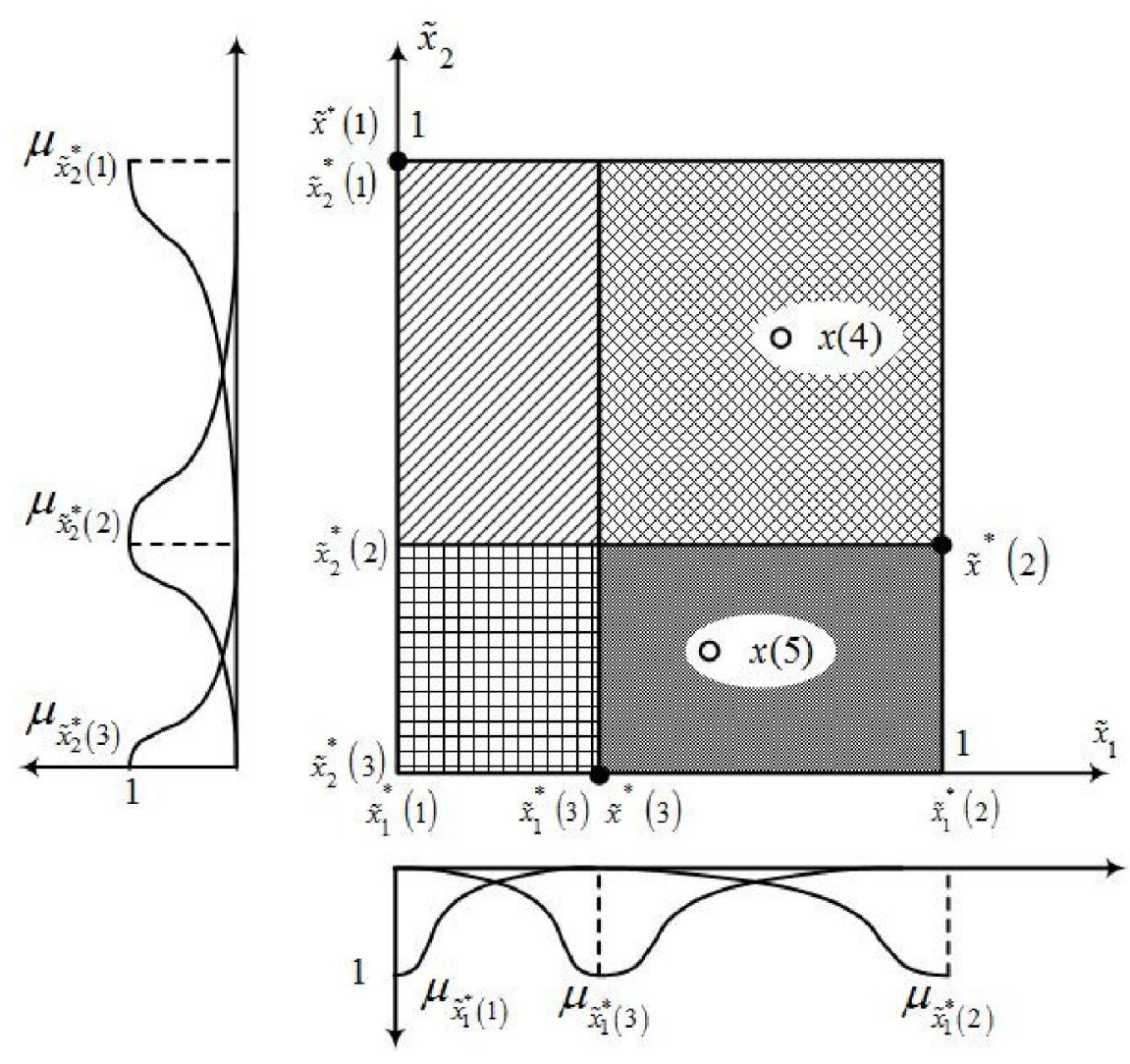

Fig. 4 - Hatched areas of the neuro-fuzzy autoassociative memory 
It is easy to see that the patient $\tilde{x}(5)$ belongs to the hatched areas of the $\tilde{x}^{*}(2)$ and $\tilde{x}^{*}(3)$ at the same time, so a "hybrid" patients signal will appear in the system output:

$$
\begin{gathered}
\overline{\tilde{x}}^{*}(\tilde{x}(5))=\mu_{\tilde{x}^{*}(2)}(\tilde{x}(5)) \cdot \tilde{x}^{*}(2)+ \\
+\mu_{\tilde{x}^{*}(3)}(\tilde{x}(5)) \cdot \tilde{x}^{*}(3) .
\end{gathered}
$$

During the system testing situation, when the capacity of fundamental memory $l$ is high or when centers of membership functions set irregularly can happens. Then attraction regions are very small or irregularly cover feature space. It leads to the occurring of gaps in object space, that is to say that in the output of the second hidden layer of architecture of Fig. 1 result of membership functions aggregation became equal to zero and these patients cannot be retrieved by neuro-fuzzy network memory.

In this case, we need to compute the distances between this patient $\tilde{x}(p)$ and all patient with specific diagnoses of the fundamental memory $\tilde{x}^{*}(k)$ using expression:

$$
\begin{gathered}
d\left(\tilde{x}^{*}(k), \tilde{x}(p)\right)=\left\|\tilde{x}^{*}(k)-\tilde{x}(p)\right\|, \\
k=1,2, \ldots, l
\end{gathered}
$$

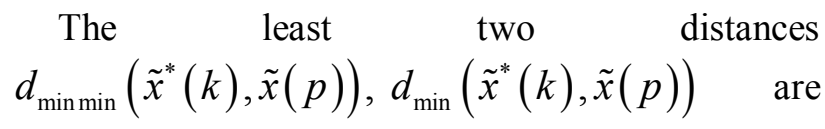
chosen, after that it's easy to compute membership levels according to the expressions [18]

$$
\begin{aligned}
& \mu^{\max \max }(\tilde{x}(p))=\frac{d_{\text {min min }}^{-2}\left(\tilde{x}^{*}(k), \tilde{x}(p)\right)}{d_{\text {min min }}^{-2}\left(\tilde{x}^{*}(k), \tilde{x}(p)\right)+d_{\text {min }}^{-2}\left(\tilde{x}^{*}(k), \tilde{x}(p)\right)}, \\
& \mu^{\max \max }(\tilde{x}(p))=\frac{d_{\text {min }}^{-2}\left(\tilde{x}^{*}(k), \tilde{x}(p)\right)}{d_{\text {min min }}^{-2}\left(\tilde{x}^{*}(k), \tilde{x}(p)\right)+d_{\text {min }}^{-2}\left(\tilde{x}^{*}(k), \tilde{x}(p)\right)} .
\end{aligned}
$$

As a result, the hybrid signal of patient occurs in the autoassociative memory output

$$
\begin{gathered}
\overline{\tilde{x}}^{*}(\tilde{x}(p))=\mu^{\max \max }(\tilde{x}(p)) \cdot \tilde{x}_{\min \min }^{*}(k)+ \\
+\mu^{\max }(\tilde{x}(p)) \cdot \tilde{x}_{\min }^{*}(k),
\end{gathered}
$$

where $\tilde{x}_{\min \min }^{*}(k), \quad \tilde{x}_{\min }^{*}(k)$ are patient from fundamental memory that are nearest to the $\tilde{x}(p)$.

\section{SIMULATION OF AUTOASSOCIATIVE MEMORY BASED ON BELL-SHAPED UNIVARIATE FUNCTIONS}

For training of autoassociative memory based on bell-shaped univariate functions the data set "pimaindians-diabets.data" [19] was used. This dataset contains 768 instances, number of attributes is 8 plus class-diagnosis (value 1 is explain as tested positive for diabetes, 500 instances, 0 - tested negative for diabetes, 268 instances). All attributes are numericvalued. The Pima Indians diabetes dataset contains 2 overlapped classes-diagnoses and for its processing we need to use fuzzy procedures.

Pattern number 183 for patients tested negative for diabetes and pattern number 207 for patients tested positive for diabetes were used as centers of each diagnosis.

The percentage of incorrectly classified patients processed by autoassociative memory that uses bellshaped univariate membership functions with tuning $\sigma$ parameter, bell-shaped univariate membership functions without tuning the one and triangle membership function are summarized in Table 1. It is easy to see that autoassociative memory that uses bell-shaped membership functions with tuning $\sigma$ parameter shows the best percentage.

Table 1. Pima Indian Diabetes classification results.

\begin{tabular}{|l|c|}
\hline $\begin{array}{c}\text { Type of bell-shaped } \\
\text { function }\end{array}$ & $\begin{array}{c}\text { Percentage of incorrectly } \\
\text { classified patients }\end{array}$ \\
\hline $\begin{array}{l}\text { with tuning } \sigma \\
\text { parameter }\end{array}$ & $35,02 \%$ \\
\hline $\begin{array}{l}\text { without tuning } \sigma \\
\text { parameter }\end{array}$ & $72,01 \%$ \\
\hline $\begin{array}{l}\text { triangle function } \\
\text { without width tuning }\end{array}$ & Impossibility of classification \\
\hline
\end{tabular}

We need to note, that in the case of triangle membership functions 178 patterns was not classified because they get in gaps between membership functions. This situation can happens using bell-shaped membership functions without tuning its width when $\sigma$ parameter is too small. 


\section{CONCLUSION}

The architecture, presented in the form of associative neuro-fuzzy memory is proposed. A principle "neurons at data points" uses for learning process of the proposed architecture. This memory is characterized by simplicity of its architecture and numerical realization. This neuro-fuzzy associative memory permits to realize the retrieval in the form of weighted combination patients of fundamental memory.

\section{REFERENCES}

[1] R. Rizzo, "Computational intelligence methods for bioinformatics and biostatistics," in Lecture Notes in Bioinformatics (7th International Meeting, CIBIB'2010), Palermo, Italy, September 16-18, 2010, Springer, 2011, 301 p.

[2] A.N. Michel, J.A. Farrel, "Associative memories via artificial neural networks," IEEE Control System Magazine, Vol. 10, Issue 3, pp. 6-17, 1990.

[3] K.-L. Du, M.N.S. Swami, Neural Networks and Statistical Learning, London: Springer-Verlag, 2014, 824 p.

[4] D.F. Specht, "Probabilistic neural networks for classification, mapping, or associative memory," in Proceedings of the IEEE International Conference on Neural Networks, 1988, Vol. 1, pp. 525-432.

[5] T. Hastie, R. Tibshirani, J. Friedman, Data Mining, Inference and Prediction, SpringerVerlag, 2009.

[6] S. Haykin, Neural Networks and Learning Machine, New York: Prentice Hall, Inc., 2009, $906 \mathrm{p}$.

[7] M.H. Hassoun, P.B. Watta, Associative Memory Networks, in "Handbook of Neural Computation" Oxford: IOP Publishing Ltd. and Oxford University Press, 1997.

[8] D.A. Simovici, C. Djeraba, Mathematical Tools for Data Mining. Set Theory, Partial Orders, Combinatorics, Springer-Verlag, 2014.

[9] J. Kacpzyk, W. Pedrycz, Handbook of Computational Intelligence, Springer-Verlag, 2015.

[10] P.P. Angelov, Handbook on Computational Intelligence, World Scientific Publishing Company Pte Limited, 2016.

[11] V. Torra, A. Dahlbom, Y. Narukawa, Fuzzy Sets, Rough Sets, Multisets and Clustering, Springer-Verlag, 2017.

[12] K.J. Cios, W. Pedrycz, Neuro-fuzzy algorithms, In "Handbook on Neural Computation". Oxford: IOP Publishing Ltd and Oxford University Press, 1997.
[13] A. Casey, Soft Computing. Developments, Methods and Applications, Nova Science Publisher Inc., 2016.

[14] L. Rutkovski, Flexible Neuro-Fuzzy Systems. Structure, Learning and Performance Evaluation, Kluwer Academic Publishers, Boston, 2004.

[15] Z. Zeng, J. Wang, Advanced in Neural Network Research and Applications, Springer-Verlag, 2010.

[16] I.N. Da Silva, D.H. Sparti, R.A. Flauzino, L.H. Batocci Liboni, S.F. dos Reis Alves, Artificial Neuron Networks. A Practical Course, Springer, 2017.

[17] Ye. Bodyanskiy, N. Teslenko, "Autoassociative neural memory based on fuzzy basis functions," Information Technology and Management Science, No. 44, pp. 9-14, 2010.

[18] F. Höppner, F. Klawonn, R. Kruse, FuzzyClusteranalyse: Verfahren für die Bilderkennung, Klassifikation und Datenanalyse, Braunschweig: Vieweg, 1996, $280 \mathrm{p}$.

[19] P.M. Murphy, D. Aha, UCI Repository of machine learning databases. [Online]. Available: http://www.ics.uci.edu/ mlearn/ MLRepository.html.

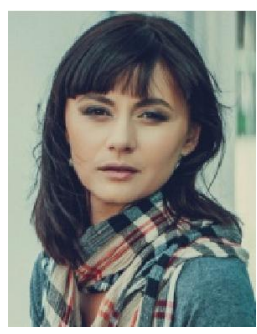

Iryna Perova graduated from Kharkiv National University of Radio Electronics in 2004. She got her PhD in 2008. She obtained an academic title of the Senior Researcher in 2015. She obtained an academic title of the Associate Professor in 2016. Ph.D. Perova is

an associate professor in Biomedical engineering department at Kharkiv National University of Radio Electronics. She has more than 40 scientific publications including one invention. Her research interests are medical data mining, systems of computational intelligence, neuro- and neo-fuzzysystems for medical diagnostics tasks, on-line systems that have to do with control, identification, clustering, diagnostics and fault detection.

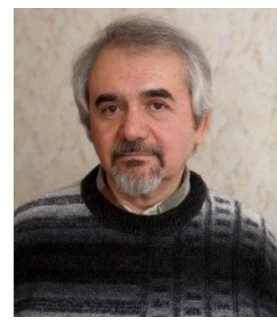

Yevgeniy Bodyanskiy. In 1971 he graduated with honour from Kharkiv National University of Radio Electronics. In 1980 he defended the Doctoral Thesis. In 1984 he was awarded the academic title of Senior Researcher. In 1990 he was awarded Dr. habil.

sc. ing. degree. In 1994 he was awarded the academic title of Professor. His major fields of research are evolving hybrid systems of 
computational intelligence, data stream mining, data science, and big data.

Since 1974 he has been working at Kharkiv National University of Radio Electronics. In 19741976 he was a Researcher; in 1977-1983 he was a Senior Researcher; in 1986-1991 he was a Scientific Head of Control Systems Research Laboratory; in 1991-1992 he was a Research Fellow. Since 1992 he is a Professor of Artificial Intelligence Department at KhNURE, Scientific Head of Control Systems Research Laboratory at
KhNURE. He has more than 660 scientific publications, including 42 inventions and 16 monographs. Research interests include hybrid systems of computational intelligence: adaptive, neuro-, wavelet-, neo-fuzzy-, real-time systems, including problems connected with control, identification, forecasting, clustering, diagnostics, fault detection in technical, economic, medical and ecological objects.

$\mathrm{He}$ is the IEEE senior member, member of 4 scientific and 7 editorial boards. 